\title{
Efecto de la cobertura vegetal de ribera en las comunidades ícticas en el bosque húmedo premontano y muy húmedo premontano del Alto Magdalena, Colombia
}

\author{
José Luis Poveda Cuellar ${ }^{1, *}$, Edwin Orlando López-Delgado ${ }^{1,2}$, Francisco A. Villa-Navarro $^{1,3}$ \\ ${ }^{1}$ Grupo de Investigación en Zoología, Facultad de Ciencias, Universidad del Tolima, Ibagué, Colombia \\ ${ }^{2}$ Estudiante Ph.D. Wildlife and Fisheries Science, Texas A\&M University, Winemiller Aquatic Lab, Texas, Estados Unidos \\ ${ }^{3}$ Laboratorio de Socioecosistemas, Departamento de Ecología, Universidad Autónoma de Madrid, España
}

\section{Resumen}

El objetivo de este estudio fue determinar la influencia de la cobertura vegetal en la composición, la estructura y la diversidad de la ictiofauna en las quebradas de las zonas de vida de los bosques húmedo premontano (bh-PM) y muy húmedo premontano (bmh-PM) del Alto Magdalena, Colombia. Para esto, se muestrearon 23 estaciones distribuidas en las coberturas vegetales de pastos, mosaico de cultivos, arbustos y bosques en las diferentes zonas de vida. En cada estación se establecieron transectos longitudinales (100 m/ancho variable); los peces se capturaron con un equipo de pesca eléctrica y una red de arrastre $(5 \mathrm{~m} \mathrm{x} 1,5 \mathrm{~m}$, ojo de malla de $0,05 \mathrm{~m})$, y se evaluaron paralelamente algunas variables ambientales. La estructura y la diversidad de la ictiofauna se calcularon mediante el uso de modelos estándar. En cuanto a la diversidad, medida con números de Hill, no hubo diferencias significativas entre las coberturas vegetales en el bh-PM. Sin embargo, en el bmh-PM la cobertura de pastos fue la más diversa. En el análisis de escalamiento multidimensional no métrico, no se evidenciaron diferencias en la estructura del ensamblaje de peces en los tipos de cobertura vegetal, sin embargo, en el análisis canónico de correspondencias se evidenció que la cobertura de dosel, la calidad de la vegetación de ribera y la elevación influyeron de manera significativa en la composición y la distribución de la ictiofauna. Si bien la cobertura de pastos presentó la mayor diversidad íctica, es un hábitat con degradación del bosque de ribera y cambios en la temperatura, idóneo para el establecimiento de especies tolerantes e invasoras. (C) 2018. Acad. Colomb. Cienc. Ex. Fis. Nat.

Palabras clave: Números de Hill; Zonas de vida; Comunidades; Cobertura vegetal; Neotrópico.

Effect of the riparian vegetation cover on fish communities in the humid premontane and very humid premontane forest in the Upper Magdalena, Colombia

\begin{abstract}
The aim of this study was to determine the influence of the vegetation cover on the composition, structure and diversity of the ichthyofauna in streams of the life zones of humid premontane (bh-PM) and very humid premontane (bmh-PM) forests in the Upper Magdalena, Colombia. To do this, 23 sampling sites were distributed in the vegetal covers of pastures, mosaic of crops, shrubs and forests in the different life zones. At each location, longitudinal transects were established (100 m/variable width), fish were captured with an electro-fishing equipment and a seine net $(5 \mathrm{~m}$ long x 1.5 height $\mathrm{m}$, mesh eye $0.05 \mathrm{~m}$ ), and, simultaneously, some environmental variables were measured. The structure and diversity of fish were calculated by using standard models. As regards diversity measured with Hill numbers, there was no significant differences between vegetal covers in the bh-PM. However, in the bmh-PM, the grass cover was the most diverse. In the Non-Metric Multidimensional Scaling Analysis, no differences were found in the structure of the fish assemblage in the types of vegetal cover, however, the Canonical Correspondence Analysis showed that the canopy cover, the quality of the riparian vegetation and the altitude significantly influence the composition and distribution of the ichthyofauna. Although grass coverage presented the greatest fish diversity, this is an ideal habitat for the establishment of tolerant and invasive species due to the degradation of the riparian forest and changes in temperature suitable for the establishment of tolerant and invasive species. (C) 2018. Acad. Colomb. Cienc. Ex. Fis. Nat.
\end{abstract}

Key words: Hill numbers; Life zone; Communities; Vegetal cover; Neotropic.

\footnotetext{
*Correspondencia:

José Luis Poveda Cuellar, josepovedaut@gmail.com, Recibido: 12 de febrero de 2018, Aceptado: 7 de mayo de 2018, Editor: Gabriel Roldán
} 


\section{Introducción}

La vegetación de ribera es aquella que se encuentra en los márgenes de los cuerpos de agua y permite la interacción entre los ecosistemas terrestre y acuático (Granados, 2006). Algunos tipos de cobertura, definidos como bosques, vegetación natural arbustiva, pastos y mosaicos de cultivos, entre otros, generan cambios físicos y químicos debido a su composición vegetal, los cuales tienen un efecto final sobre la distribución, la abundancia y la composición de especies acuáticas y otras asociadas a ellos (Biowest, 2008; Pérez, 2015).

La vegetación de ribera permite la formación de hábitats para el desove, el cuidado parental, la alimentación y la migración (Greer, et al., 2012), factores todos favorables para la colonización de peces. A pesar de su importancia, la vegetación de ribera se encuentra amenazada por la deforestación causada por prácticas agrícolas o modificaciones físicas para el uso intensivo del recurso hídrico (Sweeney, et al., 2004), lo cual afecta negativamente los ensamblajes de peces.

Dichas afectaciones se han examinado principalmente desde una perspectiva taxonómica (Bojsen \& Barriga, 2002; Pérez, 2015), ya que en estos análisis se evidencian las interacciones bióticas y abióticas presentes, así como la funcionalidad y la estabilidad de los ecosistemas (Chase, 2003), lo cual permite entender los efectos de las actividades humanas sobre la ictiofauna.

En la región del Alto Magdalena la mayoría de los estudios sobre las comunidades ícticas se ha centrado en los análisis de la diversidad y la ecología trófica y reproductiva (Bríñez, 2005; García-Melo, 2005; Zúñiga-Upegui, 2005; Castro-Roa, et al., 2007; Lozano-Zárate, et al., 2008; Albornoz \& Conde, 2014; Pareja-Carmona, et al., 2014; Montoya, 2014; Zúñiga-Upegui, et al., 2014; López-Delgado, 2015), principalmente en la zona de vida del bosque seco tropical (bs-T); se han hecho, asimismo, algunos estudios enfocados en la taxonomía y la sistemática (Villa-Navarro, et al., 2006; Taphorn, et al., 2013; GarcíaAlzate, et al., 2015; García-Melo, 2016; Provenzano \& Villa-Navarro, 2017). Sin embargo, el único estudio sobre zonas de ribera, realizado por López-Delgado (2013) en ríos del bs-T, se sugiere que la degradación de los bosques de ribera afectan negativamente la estructura y la composición de los ensamblajes ícticos al disminuir su diversidad y riqueza, y favorecer el establecimiento de especies dominantes.

Por tal motivo, el objetivo de este estudio fue determinar el efecto de las coberturas vegetales en la ictiofauna en quebradas de los bosques húmedo premontano (bh$\mathrm{PM})$ y muy húmedo premontano (bmh-PM) en los valles interandinos del Alto Magdalena en Colombia. En el estudio se plantea que las coberturas vegetales degradadas, como los pastos y los mosaicos de cultivos, afectan negativamente la composición, la estructura y la diversidad de la ictiofauna y favorecen la presencia de especies oportunistas y resistentes a ambientes hostiles.

\section{Materiales y métodos}

Área de estudio. El término zona de vida hace referencia al tipo de vegetación dominante, estrechamente relacionada con el medio físico que determina su composición y comportamiento (Holdridge, 1996). El presente estudio se desarrolló en los bosques húmedo premontano (bh-PM) y bosque muy húmedo premontano (bmh-PM) del Alto Magdalena, Colombia. Ambas zonas de vida se encuentran en la zona cafetera del departamento del Tolima y se distribuyen entre los 900 y los $2.000 \mathrm{msnm}$, con una temperatura media anual de 18 a $24{ }^{\circ} \mathrm{C}$. El bh-PM presenta una pluviosidad anual promedio de 1.000 a $2.000 \mathrm{~mm}$ y el bmh-PM, de 2.000 a $4.000 \mathrm{~mm}$, y se le considera una prolongación muy húmeda del bh-PM (Espinal, 1977). En el departamento del Tolima estas zonas de vida han sido deforestadas para el establecimiento de cultivos agrícolas y de ganadería.

Fase de campo. Los muestreos se realizaron durante el periodo de pocas lluvias (febrero, 2017) en quebradas del municipio de Ibagué, Tolima. La clasificación de los tipos de cobertura vegetal se hizo con la ayuda de imágenes satelitales y el programa ArcGIS, con lo cual se establecieron cuatro tipos de cobertura vegetal: pastos, mosaico de cultivos, bosques y arbustos, en cada zona de vida (Tabla $1 \mathrm{~S}$, https://www.raccefyn.co/index.php/raccefyn/article/download SuppFile/623/2755). Además, mediante un diseño aleatorio estratificado se seleccionaron 23 estaciones de muestreo. En el bh-PM se ubicaron ocho estaciones distribuidas en las coberturas vegetales de arbustos (2), mosaico de cultivos (3) y pastos (3) y en el bmh-PM, 16 en las coberturas vegetales de arbustos (1), bosques (4), mosaico de cultivos (5) y pastos (6) (Tabla 1, Figura 1).

Los peces se recolectaron empleando un equipo de pesca eléctrica (SAMUS, modelo $725 \mathrm{M}$ ) en dirección aguas arriba y en distintos tipos de hábitats (rápidos, remansos) y sustratos (roca, grava, arena, lodo y hojarasca), a lo largo de un transecto longitudinal de $100 \mathrm{~m}$ de largo y ancho variable, con un esfuerzo de muestreo de una hora. Además, la pesca eléctrica se complementó con tres bloqueos utilizando una

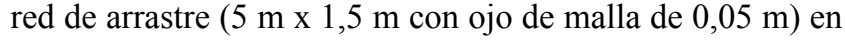
cada uno de los puntos de muestreo.

Una vez capturados, los ejemplares fueron anestesiados con eugenol, y posteriormente se fijaron en una solución de formol al $10 \%$ para su determinación taxonómica empleando diferentes claves y descripciones (Eigenman, 1912, 1922; de Pina, 1992; Buitrago, 1995; Villa-Navarro, et al., 2004; Maldonado-Ocampo, et al., 2005), trabajos de grado (Bríñez, 2004; García-Melo, 2005, 2009; Zúñiga-Upegui, 2005) y distribuciones geográficas (DoNascimiento, et al., 2017) en el Laboratorio de Investigación en Zoología (LABINZO) de la Universidad del Tolima. Es importante mencionar que se determinaron cinco morfotipos de Astroblepus: Astroblepus sp.1, Astroblepus sp.2 (Bríñez, 2004), Astroblepus sp.3 (sensu A. chotae, Bríñez, 2004), Astroblepus sp.4 (sensu A. trifasciatus, Bríñez, 2004) y Astroblepus sp.5 (sensu A. unifasciatus, Bríñez, 2004), 
Tabla 1. Estaciones de muestreo en el bosque húmedo premontano (bh-PM) y el bosque muy húmedo premontano (bmh-PM) del Alto Magdalena, Colombia. Arb=arbustos; $\mathrm{Bpt}=$ bosques; $\mathrm{Mc}=$ mosaico de cultivos; Pas=pastos.

\begin{tabular}{|c|c|c|c|c|c|c|c|}
\hline \multirow{2}{*}{$\begin{array}{l}\text { Zona de } \\
\text { vida }\end{array}$} & \multirow[t]{2}{*}{ Estación } & & \multirow[t]{2}{*}{ Sigla } & \multirow{2}{*}{$\begin{array}{c}\text { Cobertura } \\
\text { vegetal }\end{array}$} & \multirow{2}{*}{$\begin{array}{c}\text { Elevación } \\
\text { (msnm) }\end{array}$} & \multicolumn{2}{|c|}{ Coordenadas } \\
\hline & & & & & & $\mathbf{N}$ & $\mathbf{W}$ \\
\hline bh-PM & E1 & Chembe & QCHE & $\mathrm{Bpt}$ & 1300 & $4^{\circ} 28^{\prime} 45.5^{\prime \prime}$ & $75^{\circ} 10^{\prime} 03.8^{\prime \prime}$ \\
\hline bh-PM & E2 & Chembe & QCHE & $\mathrm{Mc}$ & 975 & $4^{\circ} 27^{\prime} 29.3 ”$ & $75^{\circ} 08^{\prime} 46.1$ ' \\
\hline bh-PM & E3 & Chembe & QCHE & $\mathrm{Mc}$ & 1133 & $4^{\circ} 28^{\prime} 10.8^{\prime \prime}$ & $75^{\circ} 09^{\prime} 26.3^{\prime \prime}$ \\
\hline bh-PM & $\mathrm{E} 4$ & Potrerogrande & QPG & $\mathrm{Mc}$ & 1150 & $4^{\circ} 23^{\prime} 26.6 ”$ & $75^{\circ} 12^{\prime} 38.0^{\prime \prime}$ \\
\hline bh-PM & E5 & Cocare & QCC & Arb & 1035 & $4^{\circ} 28^{\prime} 25.9^{\prime \prime}$ & $75^{\circ} 08^{\prime} 13.3^{\prime \prime}$ \\
\hline bh-PM & E6 & Cocare & QCC & Arb & 1241 & $4^{\circ} 29^{\prime} 29.1^{\prime \prime}$ & $75^{\circ} 08^{\prime} 26.6^{\prime \prime}$ \\
\hline bh-PM & E7 & Potrerogrande & QPG & Pas & 1003 & $4^{\circ} 23^{\prime} 08.6 "$ & $75^{\circ} 11^{\prime} 58.9^{\prime \prime}$ \\
\hline bh-PM & E8 & Chumba & QCHU & Pas & 975 & $4^{\circ} 48^{\prime} 55.9^{\prime \prime}$ & $75^{\circ} 05^{\prime} 36.9^{\prime \prime}$ \\
\hline bh-PM & E9 & Chumba & QCHU & Pas & 1125 & $4^{\circ} 29^{\prime} 06,2 ”$ & $75^{\circ} 05^{\prime} 48,6^{\prime \prime}$ \\
\hline bmh-PM & E10 & Los Caballos & QLC & Bpt & 1434 & $4^{\circ} 22^{\prime} 57.3^{\prime \prime}$ & $75^{\circ} 19^{\prime} 56.6^{\prime \prime}$ \\
\hline bmh-PM & E11 & San Rafael & QSR & $\mathrm{Bpt}$ & 1394 & $4^{\circ} 22^{\prime} 49.4^{\prime \prime}$ & $75^{\circ} 19^{\prime} 48.8^{\prime \prime}$ \\
\hline bmh-PM & E12 & Gonzales & QGO & $\mathrm{Bpt}$ & 1680 & $4^{\circ} 31^{\prime} 11.2 ”$ & $75^{\circ} 18^{\prime} 41.1^{\prime \prime}$ \\
\hline bmh-PM & E13 & Potrerogrande & QPG & $\mathrm{Mc}$ & 1431 & $4^{\circ} 23^{\prime} 32.6^{\prime \prime}$ & $75^{\circ} 12^{\prime} 44.0$ ' \\
\hline bmh-PM & E14 & Ambalá & QAM & $\mathrm{Mc}$ & 1502 & $4^{\circ} 28^{\prime} 48.5^{\prime \prime}$ & $75^{\circ} 12^{\prime} 38.6^{\prime \prime}$ \\
\hline bmh-PM & E15 & Río La China & RLC & $\mathrm{Mc}$ & 1590 & $4^{\circ} 32^{\prime} 30.4^{\prime \prime}$ & $75^{\circ} 07^{\prime} 50.3^{\prime \prime}$ \\
\hline bmh-PM & E16 & Río La China & RLC & $\mathrm{Mc}$ & 1750 & $4^{\circ} 32^{\prime} 36.4^{\prime \prime}$ & $75^{\circ} 07^{\prime} 56.3^{\prime \prime}$ \\
\hline bmh-PM & E17 & Aguacates & QAG & $\mathrm{Mc}$ & 1765 & $4^{\circ} 32^{\prime} 05.6^{\prime \prime}$ & $75^{\circ} 09^{\prime} 38.3^{\prime \prime}$ \\
\hline bmh-PM & E18 & Montebonito & QMB & Arb & 1677 & $4^{\circ} 22^{\prime} 39.0 "$ & $75^{\circ} 21^{\prime} 20.4^{\prime \prime}$ \\
\hline bmh-PM & E19 & Cay & QCY & Pas & 1445 & $4^{\circ} 28^{\prime} 01.0 "$ & $75^{\circ} 15^{\prime} 27.9^{\prime \prime}$ \\
\hline bmh-PM & E20 & El Gallo & QGL & Pas & 1445 & $4^{\circ} 27^{\prime} 19.6 "$ & $75^{\circ} 16^{\prime} 11.2 ”$ \\
\hline bmh-PM & E21 & Cay & QCY & Pas & 1592 & $4^{\circ} 28^{\prime} 53.5^{\prime \prime}$ & $75^{\circ} 14^{\prime} 59.5^{\prime \prime}$ \\
\hline bmh-PM & E22 & El Gallo & QGL & Pas & 1645 & $4^{\circ} 26^{\prime} 32.1^{\prime \prime}$ & $75^{\circ} 16^{\prime} 54.1^{\prime \prime}$ \\
\hline bmh-PM & E23 & El Cajón & QCJ & Pas & 1743 & $4^{\circ} 31^{\prime} 01.0$ " & $75^{\circ} 17^{\prime} 20.4^{\prime \prime}$ \\
\hline
\end{tabular}

según DoNascimiento, et al. (2017), ya que la distribución de $A$. chotae, A. trifasciatus y A. unifasciatus se restringe a las cuencas del Pacífico colombiano. Una vez determinados y contados, los ejemplares fueron incorporados a la Colección Zoológica de la Universidad del Tolima, en la sección de ictiología (CZUT-IC).

Simultáneamente, se registraron in situ algunas variables ambientales, tales como la temperatura del agua $\left({ }^{\circ} \mathrm{C}\right)$, la conductividad eléctrica $(\mu \mathrm{S} / \mathrm{cm}), \mathrm{pH}$ (unidades de $\mathrm{pH}$ ) y los sólidos totales (ppm) empleando un equipo multiparámetro (SCHOTT ${ }^{\circledR}$ handylab multi12/Set), en tanto que la velocidad del agua $(\mathrm{m} / \mathrm{s})$ se midió con un flujómetro (General Oceanics $\subset$ - modelo $2030 \mathrm{R}$ y 2030R6), así como la profundidad (m) y el ancho (m) del cauce. Como variables asociadas a la zona de ribera se calculó el índice de calidad del bosque de ribera (QBR) con la metodología propuesta por López-Delgado, et al. (2015) y, por último, la cobertura del dosel se estimó utilizando un densiómetro cóncavo (Modelo C, Forestry Suppliers Inc.), siguiendo la metodología de Braun-Blaquet (1979).

\section{Análisis de datos}

Estructura y composición. La abundancia relativa $(\mathrm{AR} \%)$ se determinó a partir del número de individuos de cada especie recolectados y su relación con el número total de individuos de la muestra. Con el fin de determinar diferencias significativas entre las abundancias de los distintos tipos de cobertura vegetal por zona de vida, se hizo un análisis de varianza (ANOVA) no paramétrico (Kruskall-Wallis, $\mathrm{p}<0,05$ ), usando la función kruskal.test en el paquete vegan del programa R (R Development Core Team, 2017).

La diversidad de la fauna íctica se evaluó utilizando el número efectivo de especies o números de Hill (Hill, 1973; Jost, 2006) mediante la función diversity del paquete vegan del programa R (R Development Core Team, 2017). La diversidad, medida en números de Hill, se calculó como el parámetro descriptivo de las relaciones especie-abundancia, con el fin de observar variaciones a nivel espacial en la estructura de los ensamblajes. Además, se emplearon curvas de estimación (interpolación y extrapolación) para comparar los tipos de cobertura vegetal y establecer diferencias 


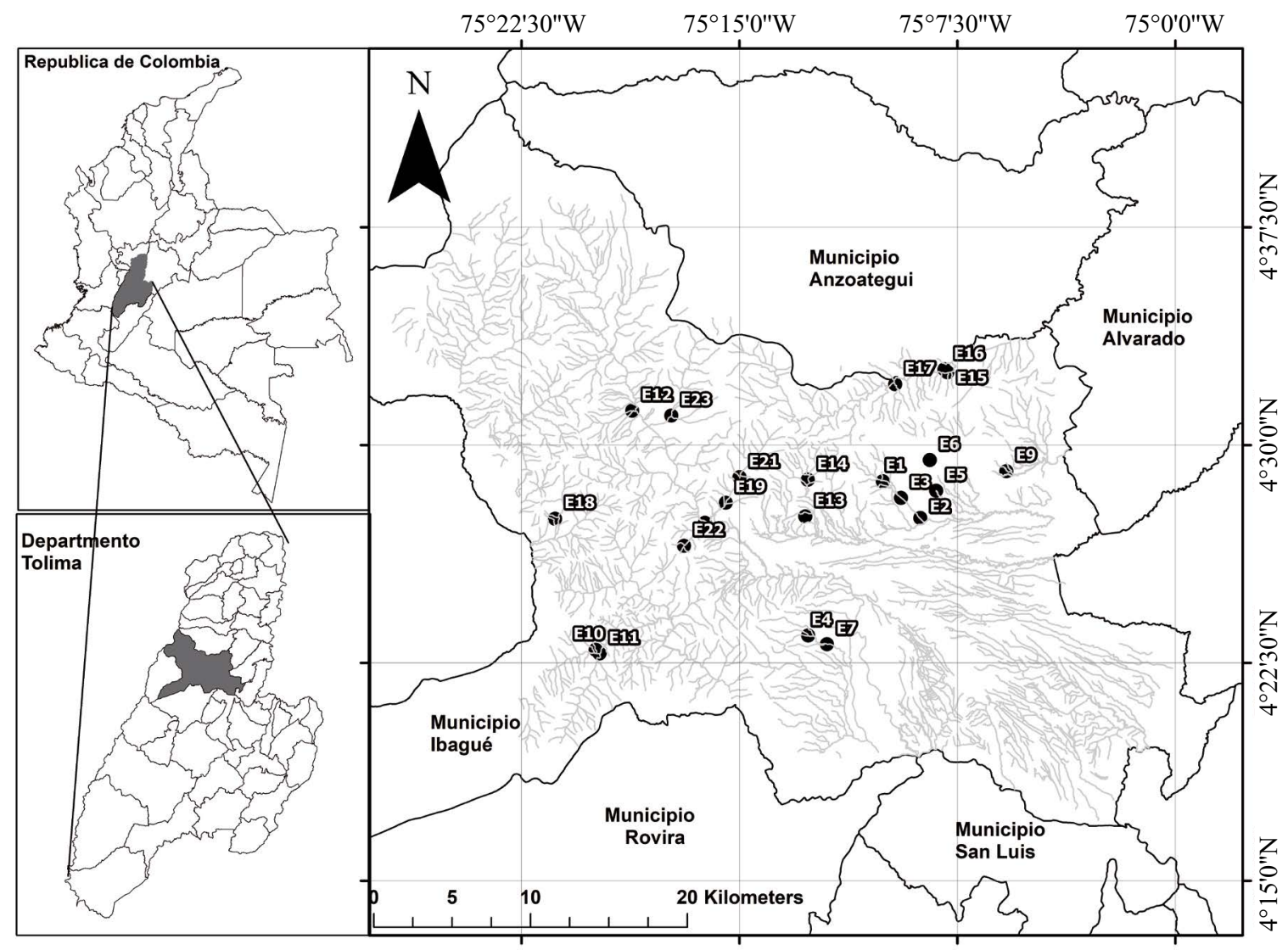

Figura 1. Estaciones de muestreo en las zonas de vida del bosque húmedo premontano (bh-PM) y el bosque húmedo muy premontano (bmh-PM) de Ibagué, Tolima

significativas por zona de vida. Estos análisis se hicieron utilizando la función iNEXT del paquete iNEXT del programa R (R Development Core Team, 2017).

Para determinar la presencia de especies indicadoras de los tipos de cobertura vegetal en cada zona de vida, se utilizó el análisis indicador de especies propuesto por Dufrêne \& Legendre (1997), utilizando la función indval del paquete indicspecies del programa R (R Development Core Team, 2017).

Por último, con el fin de determinar diferencias significativas en la estructura del ensamblaje de peces entre los tipos de cobertura vegetal por zona de vida, se hizo un análisis de escalamiento no métrico multidimensional (ENMM), acompañado de un análisis de similitud (ANOSIM) (Chapman \& Underwood, 1999), con la ayuda de la función metaMDS del paquete vegan del programa R (R Development Core Team, 2017).

Relación entre las variables ambientales y la fauna íctica. Para determinar la relación entre el ensamblaje de peces y las variables ambientales en los distintos tipos de cobertura vegetal, se hizo un análisis canónico de correspondencias (ACC), utilizando dos grupos de matrices (especies por cobertura vegetal y variables ambientales por cobertura vegetal). Las variables que influyeron significativamente $(p<0,05)$ en la estructura del ensamblaje de especies, se determinaron mediante la prueba de Monte Carlo con 999 permutaciones. Se determinaron los factores de inflación de la variancia (FIV) para reducir la multicolinealidad de las variables ambientales, y solo las variables con un factor de inflación menor de diez $(<10)$ se utilizaron en el CCA $($ Ter Braak \& Smilauer, 2002). Estos análisis se hicieron con el programa estadístico Canoco para Windows 4.5 (Braak \& Smilauer, 2002).

\section{Resultados}

Composición y estructura de la ictiofauna. Se recolectaron 1.636 individuos pertenecientes a 12 especies, siete géneros, cinco familias y tres órdenes. En el bh-PM se registraron 911 individuos, distribuidos en tres tipos de cobertura vegetal (pastos, mosaico de cultivos y arbustos), pertenecientes a tres órdenes, cinco familias y 12 especies, las cuales representan aproximadamente el $10 \%$ de las reportadas para el Alto Magdalena (Villa-Navarro, et al., 2006), el $25 \%$ de las reportadas para la cuenca del río Coello (Villa-Navarro, et al., 2004) y el $27 \%$ de las informadas para la cuenca del río Totare (Villa-Navarro, et al., 2007), estas dos últimas en el mismo rango altitudinal del área estudiada (Tabla 2S, https:// www.raccefyn.co/index.php/raccefyn/article/download SuppFile/623/2756). 
En cuanto a la distribución espacial de las especies, Astroblepus grixalvi, Astroblepus homodon, Creagrutus aff. paralacus y Trichomycterus sp.1 fueron frecuentes en los tres tipos de coberturas vegetal. Astroblepus sp.1, Astroblepus sp.3 y Astroblepus sp.4 fueron especies exclusivas de la cobertura de arbustos, y Chaestostoma cf. fisheri y Poecilia caucana, exclusivas de la cobertura de pastos (Figura 2-A). El ANOVA de Kruskall-Wallis $(\mathrm{p}=0,076)$ indicó que, con relación a la abundancia, no existen diferencias estadísticas entre los distintos tipos de cobertura vegetal del bh-PM.

Por otra parte, en el bmh-PM se registraron 725 individuos distribuidos en cuatro tipos de cobertura vegetal (pastos, bosques, mosaico de cultivos y arbustos), pertenecientes a dos órdenes, tres familias y 14 especies, las cuales representan aproximadamente el $10 \%$ de las reportadas para el Alto Magdalena (Villa-Navarro, et al., 2006), el $29 \%$ de las informadas para la cuenca del río Coello (Villa-Navarro, et al., 2004) y el $31 \%$ de las reportadas para la cuenca del río Totare (Villa-Navarro, et al., 2007), estas dos últimas en el mismo rango altitudinal del área estudiada (Tabla 3S, https://www.raccefyn.co/index.php/raccefyn/article/download SuppFile/623/2757).

En cuanto a la distribución espacial de las especies en los diferentes tipos de cobertura, Astroblepus chapmani, A. grixalvi, A. homodon, Astroblepus sp.2, Astroblepus sp.3 y Astroblepus sp.4, se registraron en dos o más tipos de cobertura vegetal. Por otra parte, Bryconamericus tolimae, Astroblepus sp.1 y Trichomycterus sp.1 fueron exclusivas de la cobertura de pastos, Astroblepus guentheri, Astroblepus sp.5 y Trichomycterus retropinis de la cobertura de mosaico de cultivos, Trichomycterus transandianus de la cobertura de bosques y Astroblepus micrecens de la cobertura de arbustos (Figura 2-B). El ANOVA de Kruskall-Wallis $(\mathrm{p}=0,089)$ indicó que, con relación a la abundancia, no existen diferencias estadísticas entre los distintos tipos de cobertura vegetal del bmh-PM.

El análisis ENMM en el bh-PM y el bmh-PM reveló que no existen diferencias entre la composición y la estructura de los distintos tipos de cobertura vegetal evaluados, lo cual fue corroborado por el ANOSIM en el bh-PM $(\mathrm{R}=0,075$, $\mathrm{p}=0,32$ ) (Figura 1S-A, https://www.raccefyn.co/index.php/ raccefyn/article/downloadSuppFile/623/2754) y en el bmh-PM $(\mathrm{R}=0,12, \mathrm{p}=0,13)$ (Figura 1S-B, https://www.raccefyn.co/ index.php/raccefyn/article/downloadSuppFile/623/2754). Por último, en el bh-PM la cobertura de pastos se caracterizó por la presencia de especies indicadoras como $C$. cf. fisheri ( $\mathrm{VI}=1,0, \mathrm{p}<0,05)$ y Trichomycterus sp. $1(\mathrm{VI}=0,8, \mathrm{P}<0,05)$ y la de arbustos por la presencia de Astroblepus sp.1 $(\mathrm{VI}=1,0, \mathrm{p}<0,05)$. En el bmh-PM no se registraron especies indicadoras para ningún tipo de cobertura.

Diversidad. En la zona de vida del bh-PM el tipo de cobertura con el mayor número efectivo de especies en los tres órdenes evaluados fue el de pastos, seguido por el de arbustos y el mosaico de cultivos. Sin embargo, según los resultados de los intervalos de confianza de las curvas de rarefacción e interpolación, no se observaron diferencias significativas en la diversidad de las coberturas evaluadas (Figura 3)

De forma similar, en la zona de vida de bmh-PM el número efectivo de especies más alto en los tres órdenes evaluados se registró en la cobertura de pastos, seguida por la de bosques, mosaico de cultivos y arbustos. Los intervalos de confianza de las curvas de interpolación y extrapolación mostraron diferencias en la diversidad en las coberturas evaluadas, principalmente entre la de pastos y la de mosaico de cultivos (Figura 4).

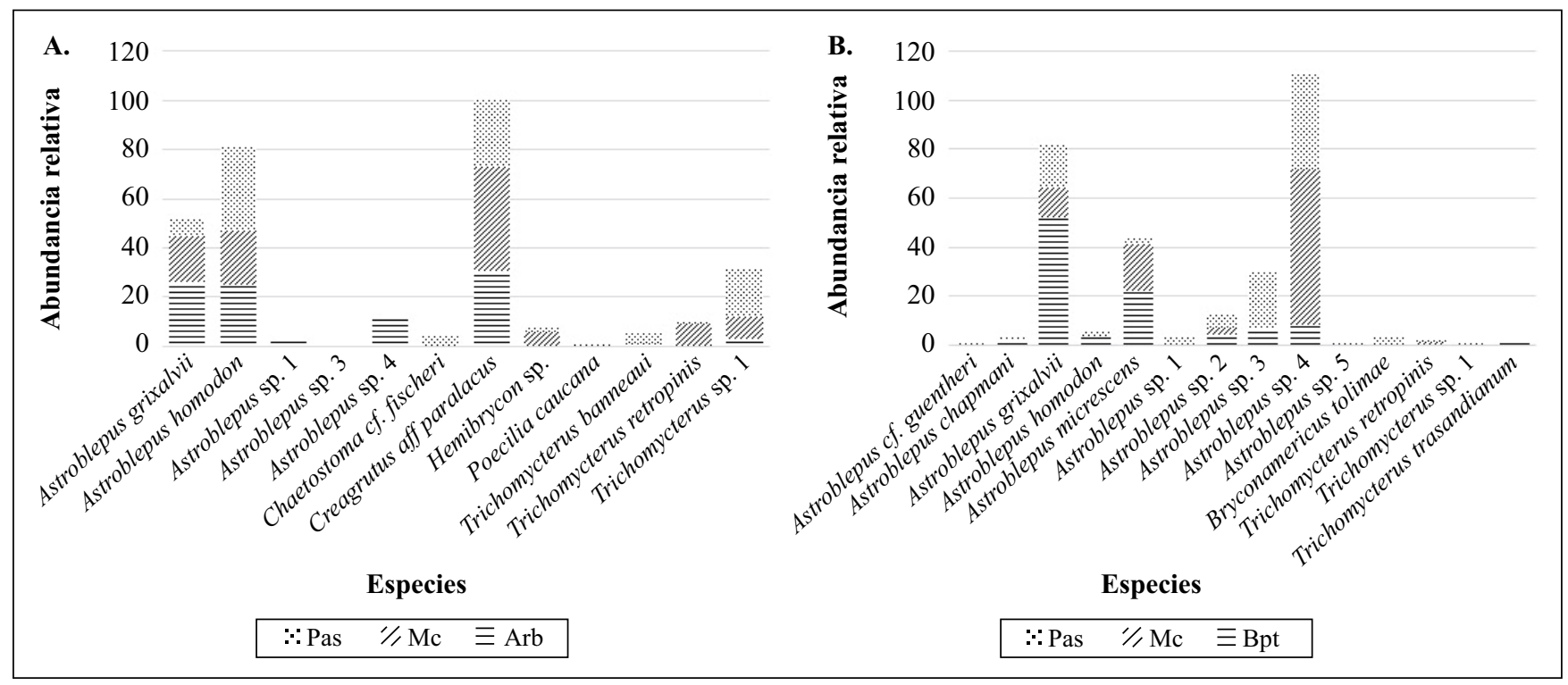

Figura 2. Abundancia relativa de las especies registradas en las coberturas vegetales de pastos, mosaico de cultivos, bosques y arbustos. A. Bosque húmedo premontano (bh-PM). B. Bosque muy húmedo premontano (bmh-PM) del municipio de Ibagué. 


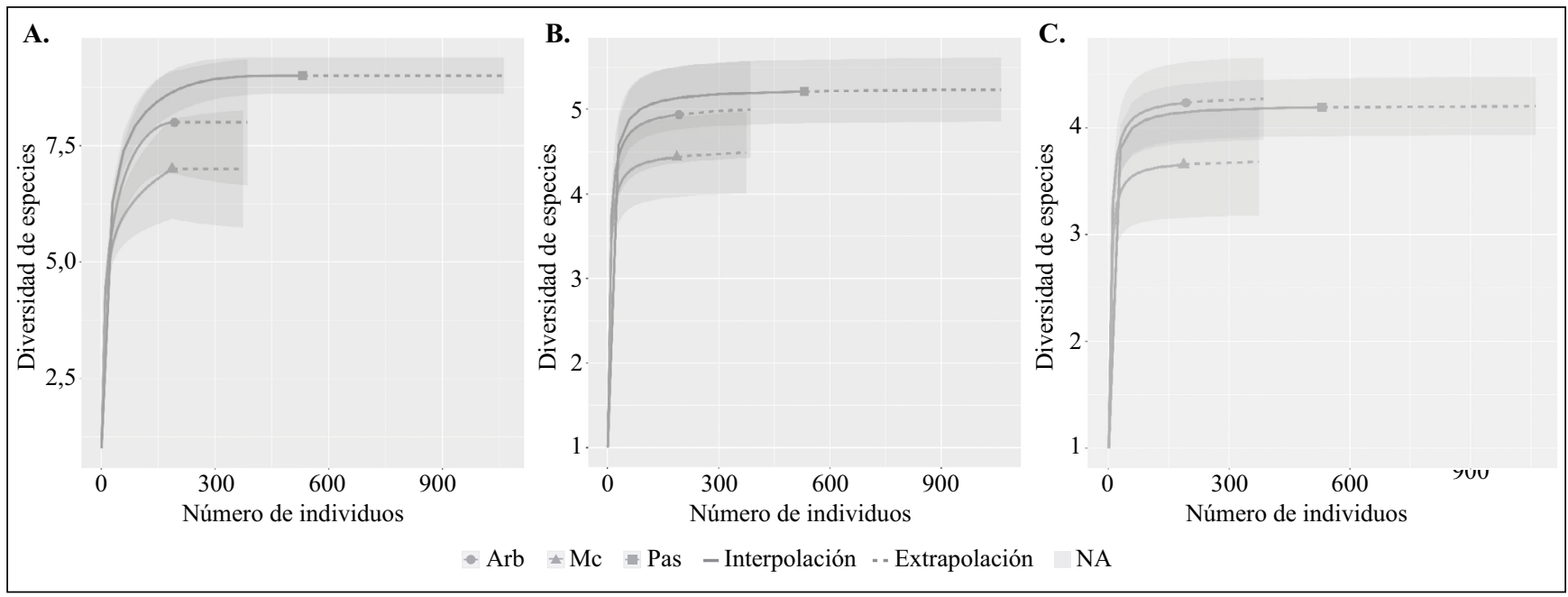

Figura 3. Diversidad de especies de peces en los distintos tipos de cobertura vegetal del bosque húmedo premontano (bh-PM) del municipio de Ibagué. A. Riqueza de especies (diversidad de orden $q=0$ ). B. Diversidad de orden $q=1$. C. Diversidad de orden: $q=2$.

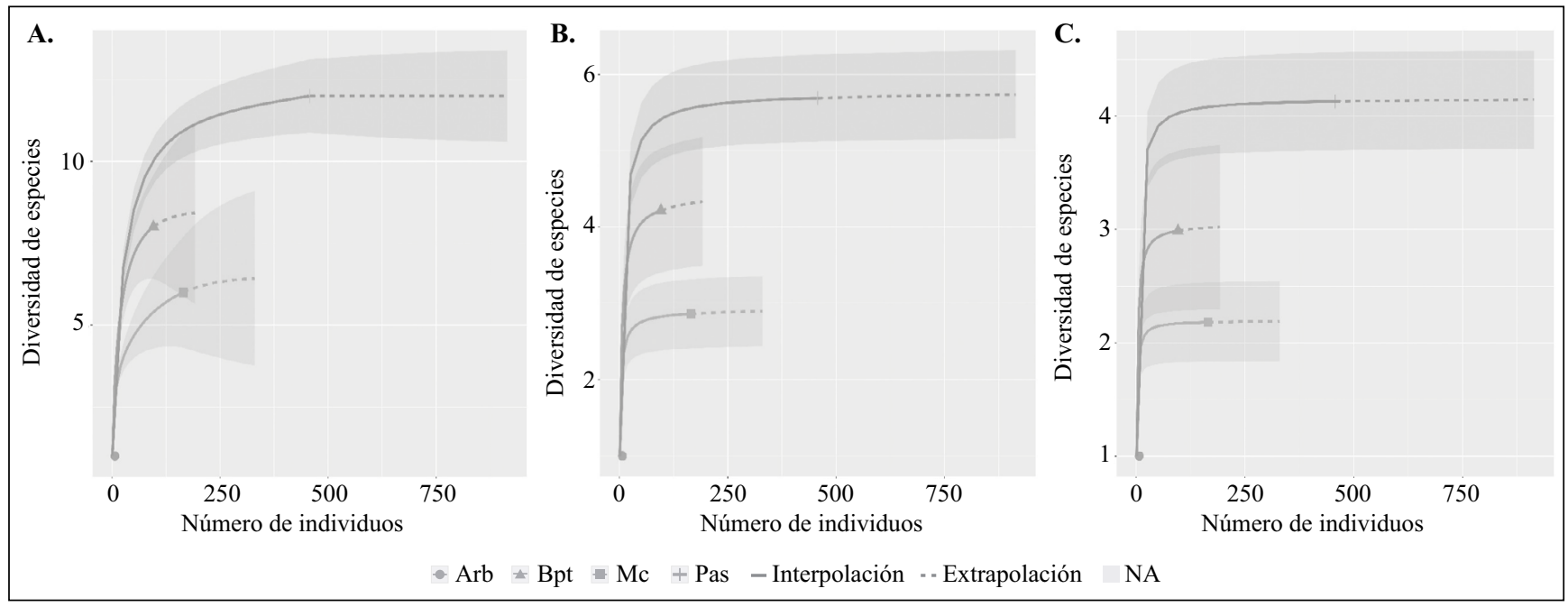

Figura 4. Diversidad de especies de peces en los distintos tipos de cobertura vegetal del bosque muy húmedo premontano (bmh-PM) del municipio de Ibagué. A. Riqueza de especies (diversidad de orden: $q=0$ ). B. Diversidad de orden: $q=1$. C. Diversidad de orden: $q=2$.

Ensamblaje de peces y tipos de cobertura vegetal. En el ACC del bh-PM los dos primeros ejes explicaron el $72 \%$ de la variación de los datos, la única variable que influyó de forma significativa en la composición y la estructura de la ictiofauna fue la cobertura del dosel $(\mathrm{p}<0,05)$. Esta variable se asoció positivamente con la estación QCC (1.241 msnm) en la cobertura de arbustos y con Astroblepus sp.1, Astroblepus sp.3 y Astroblepus sp.4, y negativamente con las estaciones QPG (1.003 m snm) en la cobertura de pastos, con la QPG $(1.150 \mathrm{msnm})$ y la QCHE (1.133 $\mathrm{msnm})$ en la cobertura de mosaico de cultivos y con $C$. cf. fisheri, $C$. aff. paralacus, Hemibrycon sp., P. caucana y T. retropinis (Figura 5).

Por último, en el ACC del bmh-PM los dos primeros ejes explicaron el $56 \%$ de la variación, y las variables que influyeron significativamente en la composición y la estructura de la ictiofauna fueron la elevación y la calidad de la vegetación de la ribera $(\mathrm{QBR})(\mathrm{p}<0,05)$. En el eje 1, la variable de elevación se asoció positivamente con las estaciones QMB (1.677 msnm) en la cobertura de arbustos, QGO (1.680 msnm) en la cobertura de bosques, QAG (1.765 msnm), RLC (1.590 msnm), y con la RLC (1.750 msnm) en la cobertura de mosaico de cultivos, QCJ (1.749 $\mathrm{msnm})$ y la QCY (1.745 msnm) en la cobertura de pastos y A. micrecens y Astroblepus sp.5. Las estaciones QCY (1.445 msnm) y QGL (1.445 msnm) en la cobertura de pastos, y QSR (1.394 msnm) en la cobertura de bosques, se asociaron negativamente con la elevación, al igual que con A. homodon, Astroblepus sp.2, Astroblepus sp.3, B. tolimae y Trichomycterus sp.1 (Figura 6).

Asimismo, en el eje 1 el QBR se asoció positivamente con las estaciones QLC (1.434 msnm) y QGO (1.680 msnm) 
en la cobertura de bosques, y con la QMB (1.677 msnm) en la cobertura de arbustos, y con $T$. transandianus, y negativamente con la QCY (1445 m snm) y la QGL (1445 m snm) en la cobertura de pastos y la QSR (1394 m snm) con la cobertura de bosques y con A. homodon, Astroblepus sp.2, Astroblepus sp.3, B. tolimae y Trichomycterus sp.1 (Figura 6).

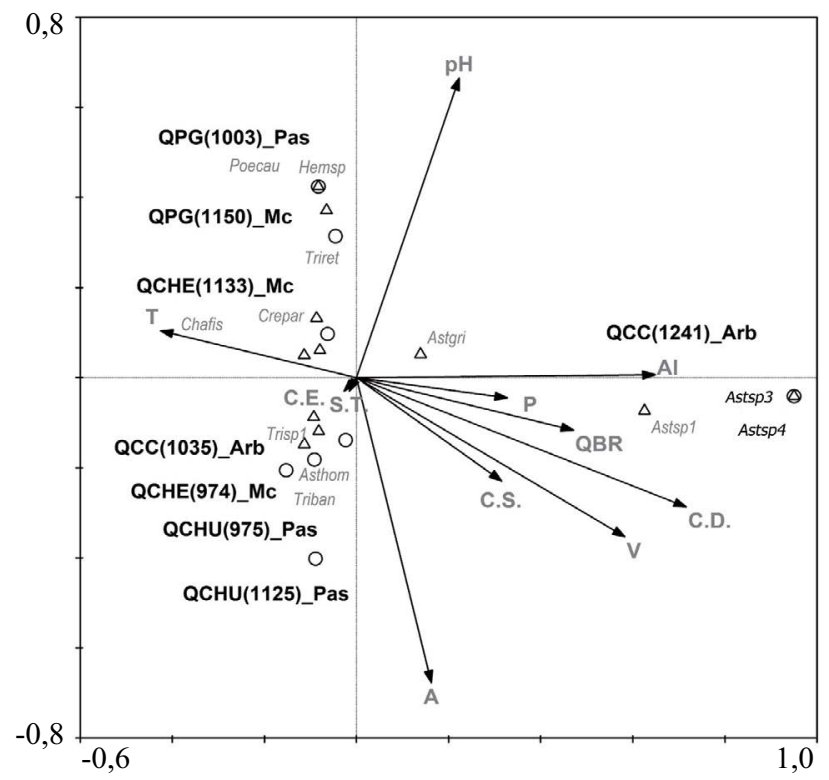

Figura 5. Diagrama trilineal (triplot) del análisis canónico de correspondencias (ACC) de los distintos tipos de cobertura vegetal, las variables ambientales y el ensamblaje de peces del bosque húmedo premontano (bh-PM) en el municipio de Ibagué

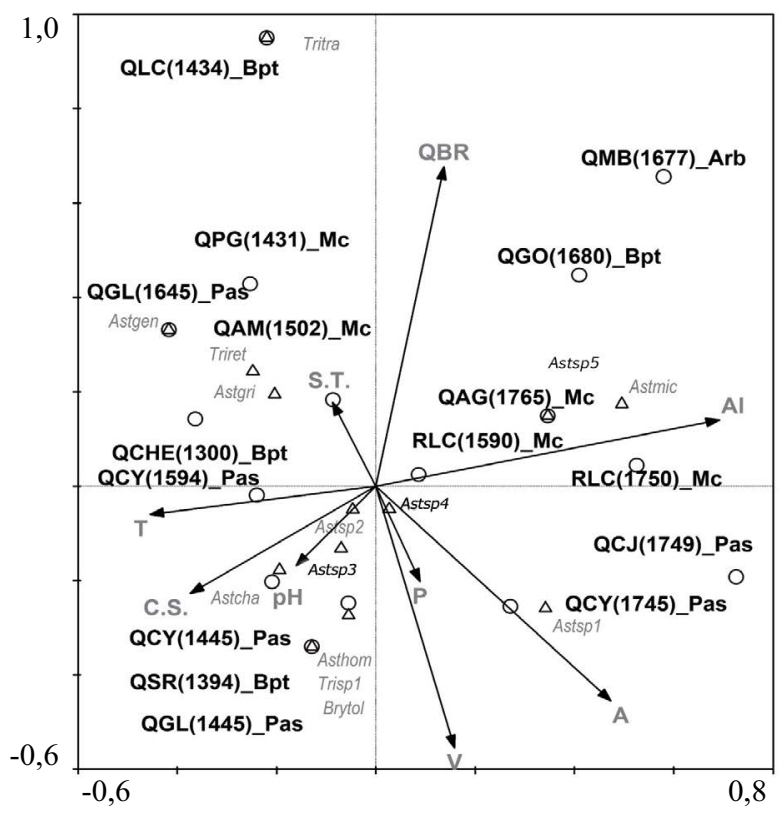

Figura 6. Diagrama trilineal (triplot) del análisis canónico de correspondencias de los distintos tipos de cobertura vegetal, las variables ambientales y el ensamblaje de peces del bosque muy húmedo premontano (bmh-PM) en el municipio de Ibagué

\section{Discusión}

Estructura y composición. La ausencia de diferencias en la estructura y composición de los ensamblajes de peces entre los tipos de cobertura vegetal, tanto en el bh-PM como en el bmh-PM, puede atribuirse al hecho de que las especies halladas son generalistas o toleran amplios márgenes de variación en la oferta de recursos en los distintos hábitats. En el bh-PM, $C$. aff. paralacus registró los valores más altos de abundancia en las estaciones ubicadas en coberturas de pastos y arbustos, algunas de las cuales (QCHU y QPG) estaban rodeadas por arbustos y árboles que, probablemente, ofertaron semillas e insectos, recursos usualmente aprovechados por esta especie (Lozano-Zárate, 2008).

La cobertura de pastos se considera un ecosistema terrestre dinámico expuesto a diferentes perturbaciones (quema, herbivoría y sequía) (Velázquez \& Gómez, 2009), que limita el aporte de recursos a los ecosistemas acuáticos que rodea, favoreciendo la presencia de especies resistentes y de amplia dispersión, como es el caso de P. caucana, la cual, por sus rasgos de historia de vida (ovoviparidad, tolerancia a bajos niveles de oxígeno disuelto y a altas temperaturas), soporta las presiones de ambientes fluctuantes (Casatti, et al., 2009; Gordon, et al., 2009; Martinez, et al., 2016). Esto también ocurriría con $C$. cf. fisheri, ya que algunas especies de la familia Loricariidae presentan tolerancia a variaciones e impactos ambientales como la disminución del oxígeno, y presentan hábitos alimenticios relacionados con el consumo de algas perifíticas, las cuales se ven favorecidas por el incremento de la intensidad lumínica y de la temperatura causado por la disminución de la vegetación de ribera (Kennard, et al., 2005; Vieira \& Shibatta, 2007).

En el bmh-PM al parecer sucede lo mismo con Astroblepus sp.4, la cual registró su mayor abundancia en la estación QCY (pastos) caracterizada por su fondo de arena y sus recursos autóctonos. Los macroinvertebrados acuáticos (Ephemerotera y Diptera) son consumidos frecuentemente por esta especie (Bríñez, 2004).

Diversidad. La ausencia de diferencias significativas en los valores de riqueza $(\mathrm{q}=0)$, diversidad $(\mathrm{q}=1)$ y dominan-cia $(\mathrm{q}=2)$ en los tres tipos de cobertura vegetal del bh-PM podría indicar que éstas proporcionaron un número similar de microhábitats (rocas, guijarro, arena, hojarasca y lodo), fuentes alimenticias (macroinvertebrados acuáticos y perifiton) y condiciones ambientales similares en las coberturas, lo que permite la formación de ensamblajes similares.

La variación en los valores de los órdenes de diversidad en los distintos tipos coberturas del bmh-PM, especialmente la diferencia en la riqueza en las coberturas conservadas (arbustos y bosques) y las degradadas (mosaico de cultivos y pastos), podría indicar que algunos ensamblajes estarían dominados por especies con capacidad de explotar recursos autóctonos, como en el caso de los mosaicos de cultivos y los pastos, y en otros donde serían frecuentes especies insectívoras y omnívoras que requieren recursos alóctonos, como en el caso de los arbustos y los bosques (Bojsen \& Barriga, 
2002), situación que se observa en arroyos del Neotrópico de zonas degradadas por la deforestación donde se registran valores altos de riqueza (Lorion \& Kennedy, 2009).

El hecho de que los pastos y los bosques registraran los valores más altos en los órdenes $\mathrm{q}=1 \mathrm{y} \mathrm{q}=2$, en tanto que en los mosaicos de cultivos se obtuvieran valores menores, podría deberse a que la diversidad varía según las condiciones ambientales (Thioulouse \& Chessel, 1992) y la complejidad del hábitat (Gregory, et al., 1991). Al parecer en los pastos y los bosques se presenta una zona de amortiguación de vegetación de ribera boscosa que ofrece un ambiente menos hostil y una mayor diversidad de material alóctono, lo cual favorece una mayor oferta de microhábitats potenciales (Pusey \& Arthington, 2003), cuya gran diversidad se asocia con la presencia de vegetación de ribera y a una gran diversidad de especies (Gregory, et al., 1991).

Por el contrario, la poca diversidad en los mosaicos de cultivos se debe a la inestabilidad de los microclimas (Correa, et al. 2015; Shimano \& Juen, 2016), a lo que habría que sumar los procesos de erosión que llevan a la sedimentación (Mercer, et al. 2014), la disminución de la cobertura de dosel (Fitzherbert, et al., 2008) y sus efectos negativos sobre el suelo y los ecosistemas acuáticos (Allan, 2004). Asimismo, los plaguicidas y fertilizantes asociados a las distintas prácticas agrícolas también están relacionados con los cambios en la concentración de nutrientes y la pérdida de diversidad (Beltrão, et al., 2009).

Ensamblaje de peces y tipos de cobertura vegetal. En la cobertura de arbustos del bh-PM las variables de cobertura del dosel, calidad de la vegetación de ribera (índice QBR), baja temperatura y mayor velocidad del agua, características de ecosistemas conservados, influyeron en la distribución de las especies (Casatti, et al., 2012). Esto podría explicar la presencia de Astroblepus sp.3 (sensu A. chotae) y Astroblepus sp.4 (sensu A. trifasciatus), las cuales dependen de recursos alóctonos como la hojarasca, las ramas y los insectos que son proporcionados por ambientes más estables (Lorion \& Kennedy, 2009; Ferreira, 2010).

Las coberturas de pastos y mosaicos de cultivos se caracterizaron por una baja cobertura de dosel, mayor temperatura, y menor velocidad y profundidad del agua, condiciones propias de ambientes degradados (Casatti, $\boldsymbol{e t}$ al., 2012), lo que favorece la presencia de $C$. cf. fisheri y $P$. caucana. La baja cobertura de dosel permite una mayor entrada de la luz en el cuerpo de agua, lo que lleva a un incremento de la diversidad y la abundancia del perifiton (Burcham, 1988; Posada, et al., 2000), recurso alimenticio requerido al menos por $C$. cf. fisheri (Zúñiga-Upegui, 2005).

En el bmh-PM la elevación sobre el nivel del mar influyó significativamente en la distribución de las especies. La geomorfología y el clima tienen efectos sobre las dinámicas de los ensamblajes de peces. Los cuerpos de agua ubicados en zonas altas se caracterizan por presentar corrientes rápidas, sustratos de roca y grava, y poca o ninguna macrófita acuática, lo que induce la presencia de especies con adaptaciones que les permitan mantenerse en estos ecosistemas (Winemiller, 2007). Esto explicaría la presencia de $A$. micrecens en estaciones del bmh-PM ubicadas en elevaciones superiores a los $1.700 \mathrm{msnm}$; esta especie ha sido reportada hasta los $2.000 \mathrm{msnm}$, aproximadamente (Cortolima, 1996; Bríñez, 2004), y se caracteriza por poseer un disco oral ventral, mandíbulas especializadas para la fijación en aguas rápidas y una región pélvica que le permite escalar rocas y cascadas, facilitando la colonización de diferentes ambientes y la exploración de una gran diversidad de recursos alimenticios y físicos (Lujan \& Armbruster, 2012; De Crop, et al., 2013; Zuluaga, et al., 2016).

\section{Conclusiones}

La ausencia de diferencias en la composición, la estructura y la diversidad entre las coberturas vegetales del bh-PM, se deben a una oferta similar de los tipos de microhábitats, de las fuentes de alimentación y de las condiciones fisicoquímicas. A pesar de esto, las coberturas vegetales degradadas, como los pastos, se caracterizan por la presencia de $P$. caucana, especie introducida en el área de estudio tolerante a los aumentos en la temperatura del agua y común en hábitats degradados.

En el bmh-PM los cambios de composición y diversidad entre las coberturas vegetales se deben al efecto de la elevación en la ictiofauna. Es importante mencionar que las zonas conservadas, como las de bosque, se caracterizaron por la presencia de especies exclusivas como T. transandianum, debido a que proveen los recursos físicos requeridos (fondos de arena) y un microclima más estable.

\section{Información suplementaria}

Tabla 1S. Caracterización de los tipos de cobertura vegetal en el bosque húmedo premontano (bh-PM) y el bosque muy húmedo premontano (bmh-PM) del Alto Magdalena, Colombia. Arb=arbustos; $\mathrm{Bpt}=$ bosques; $\mathrm{Mc}=$ mosaico de cultivos; $\mathrm{Pas}=$ pastos (Cortolima, 2012). Vea la tabla 1S en: https://www.raccefyn.co/ index.php/raccefyn/article/downloadSuppFile/623/2755

Tabla 2S. Composición y estructura de la fauna íctica registrada en las estaciones evaluadas en el bosque húmedo premontano (bh-PM) del municipio de Ibagué. Arb=arbustos; Bpt=bosques; $\mathrm{Mc}=$ mosaico de cultivos; Pas=pastos; AR: abundanca relativa. Vea la tabla $2 \mathrm{~S}$ en: https://www.raccefyn.co/index.php/raccefyn/article/ downloadSuppFile/623/2756

Tabla 3S. Composición y estructura de la fauna íctica registrada en las estaciones evaluadas en el bosque muy húmedo premontano (bmh-PM) del municipio de Ibagué. Arb=arbustos; Bpt=bosques; $\mathrm{Mc}=$ mosaico de cultivos; Pas=pastos; AR: abundanca relativa. Vea la tabla 3S en: https://www.raccefyn.co/index.php/raccefyn/article/ downloadSuppFile/623/2757

Figura 1S. Diagrama de ordenación ENMM basado en las abundancias de las especies de peces registradas en los tipos de cobertura vegetal de Ibagué. A. Bosque húmedo premontano (bh-PM). B. Bosque muy húmedo premontano (bmh-PM) del municipio de Ibagué. Vea la figura $1 \mathrm{~S}$ en: https://www.raccefyn.co/ index.php/raccefyn/article/downloadSuppFile/623/2754 


\section{Agradecimientos}

Agradecemos el apoyo financiero del Grupo de Investigación en Zoología de la Universidad del Tolima.

\section{Contribución de los autores}

Los datos usados para la elaboración de este manuscrito, hacen parte del proyecto de grado para optar al título de biólogo de José Luis Poveda Cuellar en la Universidad del Tolima, el cual fue dirigido por Francisco Antonio Villa Navarro y co-dirigido por Edwin Orlando López Delgado.

José Luis Poveda Cuellar: Fase de campo, colecta, determinación taxonómica, y redacción del manuscrito. Francisco Antonio Villa Navarro: Diseño del muestreo, Fase de campo, Ajustes estadísticos, redacción y diseño. Edwin Orlando López Delgado: Fase de campo, Análisis de datos y ajustes de redacción en discusión y resultados.

\section{Conflicto de intereses}

Los autores del presente manuscrito declaran no tener ningún conflicto de intereses.

\section{Referencias}

Albornoz, J., Conde, C. 2014. Diversidad y relaciones ecomorfológicas de la comunidad íctica de la cuenca del río Alvarado. (Tesis de Pregrado). Universidad del Tolima, Facultad de Ciencias, Departamento de Biología, Ibagué.

Allan, J.D. (2004). Landscapes and riverscapes: The influence of land use on stream ecosystems. Annual Review of Ecology, Evolution, and Systematica. 35: 257-284.

Beltrão, G. B., Medeiros, E.S., Ramos, R.T. (2009). Effects of riparian vegetation on the structure of the marginal aquatic habitat and the associated fish assemblage in a tropical Brazilian reservoir. Biota Neotropica, 9 (4): 37-43. Fecha de consulta: 10 de dicembre de 2017. Disponible en: http://www.biotaneotropica.org.br/v9n4/en/ abstract?article+bn00709042009

Bio-west, Inc. (2009). Diamond Fork and Sixth Water Creeks Riparian Vegetation and Ladies'-tresses. North Logan, Utah, Utah Reclamation Mitigation and Conservation Commission. USA.

Bojsen, B. H., Barriga, R. (2002). Effects of deforestation on fish community structure in Ecuadorian Amazon streams. Freshwater Biology. 4: 2246-2260.

Buitrago-Suárez, U. (1995). Sistemática de las especies colombianas del género Astroblepus Humboldt, 1805 (Pisces: Silurodei: Astroblepidae). (Tesis de Maestría). Universidad Nacional de Colombia, Instituto de Ciencias Naturales, Bogotá, D.C.

Braak, C. J., Smilauer, P. (2002). CANOCO Reference manual and CanoDraw for Windows user's guide: Software for Canonical Community Ordination (version 4.5). Ithaca, Microcomputer Power, p. 500.

Braun-Blanquet, J. (1979). Fitosociología. Bases para el estudio de las comunidades vegetales. Barcelona, Blume, p. 16-500.

Briñez, G.N. (2004). Distribución altitudinal, diversidad y algunos aspectos ecológicos de la familia Astroblepidae (Pisces: Siluriformes) en la cuenca del río Coello (Tolima). (Trabajo de Pregrado). Universidad del Tolima, Facultad de Ciencias, Departamento de Biología, Ibagué.
Burcham, J. (1988). Fish communities and environmental characteristics of two lowland streams in Costa Rica. Revista de Biología Tropical. 36: 273-285.

Casatti, L., Barreto, T., Gonçalves-Souza, T., Bessa, E., Manzotti, A., Silva, C., Oliveira, O. (2012). From forests to cattail: How does the riparian zone influence stream fish? Neotropical Ichthyology. 10 (1): 205-214.

Casatti, L., Ferreira, C., Carvalho, F. (2009). Grass dominated stream sites exhibit low fish species diversity and dominance by guppies: An assessment of two tropical pasture river basins. Hydrobiologia. 632 (1): 273-283.

Castro-Roa, D. (2006). Composición y estructura de la comunidad de Characiformes en la cuenca del río Prado. (Trabajo de Pregrado). Universidad del Tolima, Facultad de Ciencias, Departamento de Biología. Ibagué.

Chase, J. M. (2003). Community assembly: When should history matter? Oecologia. 136 (4): 489-498.

Correa, F., Juen, L., Rodrigues, L., Silva-Filho, H., SantosCosta, M. (2015). Effects of oil palm plantations on anuran diversity in the eastern Amazon. Animal Biology. 65: $321-335$

Cortolima. (1996). Inventario de la fauna silvestre en las cuencas de los ríos Combeima, Toche y Tochecito del municipio de Ibagué, Tolima. Corporación Autónoma del Tolima, Ibagué. p. 57.

De Crop, W., Pauwels, E., Van Hoorebeke, L., Geerinckx, T. (2013). Functional morphology of the Andean climbing catfishes (Astroblepidae, Siluriformes): Alternative ways of respiration, adhesion, and locomotion using the mouth. Journal of Morphology. 274 (10): 1164-1179.

de Pinna, M. C. (1992). A new subfamily of Trichomycteridae (Teleostei, Siluriformes), lower loricarioid relationships and a discussion on the impact of additional taxa for phylogenetic analysis. Zoological Journal of the Linnean Society. 106: 175-229.

DoNascimiento, C., Herrera-Collazos, E., Herrera-R, G., Ortega-Lara, A., Villa-Navarro, F., Usma, J., MaldonadoOcampo, J. (2017). Checklist of the freshwater fishes of Colombia: A Darwin Core alternative to the updating problem. ZooKeys. 708: 25-138

Dufrêne, M., Legendre, P. (1997). Species assemblages and indicator species: The need for a flexible asymetrical approach. Ecol. Monogr. 67: 345-366.

Eigenmann, C. H. (1912). Some results from an ichthyological reconnaissance of Colombia, South America. Indiana University Studies. 8: 1-2.

Eigenmann, C. H. (1922). The fishes of the Northwestern South America, part I. The fresh-water fishes of Northwestern South America, including Colombia, Panamá, and Pacific slopes of Ecuador y Peru, together with an appendix upon the fishes of the ríver Meta in Colombia. Mem. Carnegie Mus. 9 (1): 1-346.

Espinal, S. (1977). Zonas de vida o formaciones vegetales de Colombia. Bogotá', Colombia: Instituto Geográfico Agustín Codazzi. p. 238.

Ferreira, C., Casatti, L. (2006). Influência da estrutura do hábitat sobre a ictiofauna de um riacho em uma micro-bacia de pastagem, São Paulo, Brasil. Revista Brasileira de Zoologia. 23 (3): 642-651.

Fitzherbert, E., Struebig, M., Morel, A., Danielsen, F., Brühl, C., Donald, P., Phalan, B. (2008). How will oil palm expansion affect biodiversity? Trends in Ecology \& Evolution. 23 (10): 538-545. 
García-Melo, L. (2005). Distribución, diversidad y ecología básica de la familia Trichomycteridae (Ostariophysi: Siluriformes) en la cuenca del río Coello departamento del Tolima. (Tesis de Pregrado). Universidad del Tolima, Facultad de Ciencias, Departamento de Biología, Ibagué.

García-Melo, L. (2009). Relaciones filogenéticas de Trichomycterus (siluriformes: trichomycteridae) en el Alto Magdalena. (Trabajo de Maestría). Universidad del Tolima, Facultad de Ciencias, Departamento de Biología, Ibagué.

García-Melo, L., Villa-Navarro, F., Donascimiento, C. (2016). A new species of Trichomycterus (Siluriformes: Trichomycteridae) from the upper río Magdalena basin, Colombia. Zootaxa. 4117: 226-40.

García-Alzate, C., Taphorn, D., Roman-Valencia, C., Villa-Navarro, F. (2015). Hyphessobrycon natagaima (Characiformes: Characidae), una nueva especie para Colombia, con clave para las especies de Hyphessobrycon de la Cuenca del Magdalena. Caldasia. 37: 221-232.

Gordon S.P., Reznick, D.N., Kinnison, M.T., Bryant, M.J., Weese, D.J., Räsänen, K., Millar, N.P, Hendry, A.P. (2009). Adaptive Changes in Life History and Survival following a New Guppy Introduction. The American Naturalist. 174 (1): 34-45.

Granados, D., Hernández-García, M., López-Ríos, G. (2006). Las zonas ribereñas. Revista Chapingo Serie Ciencias Forestales y del Ambiente. 12 (1): 55-69.

Greer, M., Closs, G.P., Crow, S., Hicks, A. (2012). Complete versus partial macrophyte removal: The impacts of two drain management strategies on freshwater fish in lowland New Zealand streams. Ecology Freshwater Fish. 21: 510-520.

Gregory, S.V., Swanson, F. J., McKee, W. A., Cummins, K. W. (1991). An Ecosystem Perspective of Riparian Zones. Bioscience. 41: 540-551.

Hill, M. (1973). Diversity and evenness: A unifying notation and its consequences. Ecology. 54 (2): 427-432.

Holdridge, L. (1996). Ecología basada en zonas de vida. San José, Costa Rica: Instituto Interamericano de Cooperación para la Agricultura, IICA. San José de Costa Rica.

Jost, L. (2006). Entropy and diversity. Oikos. 113 (2): 363-375.

Kennard, M., Arthington, A., Pusey, B., Harch. B. (2005). Are alien fish a reliable indicator of river health? Freshwater Biology. 50 (1): 174-193.

López-Delgado, E., Vásquez-Ramos, F., Villa-Navarro, F., Reinoso, G. (2015). Evaluación de la calidad del bosque de ribera utilizando un método simple y rápido en dos ríos de bosque seco tropical (Tolima, Colombia). Revista Tumbaga. 1 (10): 6-29.

Lorion, C., Kennedy, B. (2009). Riparian forest buffers mitigate the effects of deforestation on fish assemblages in tropical headwater streams. Ecological Society of America. 19(2): 468-479.

Lozano- Zárate, Y. (2008). Diversidad, distribución, abundancia y ecología de la familia Characidae (Ostariophysi: Characiformes) en la cuenca del río Totare (TolimaColombia). (Tesis de Pregrado). Universidad del Tolima, Facultad de Ciencias, Ibagué.

Lujan, N., Armbruster, J. (2012). Morphological and functional diversity of the mandible in suckermouth armored catfishes (Siluriformes: Loricariidae). Journal of Morphology. 273 (1): 24-39.
Maldonado-Ocampo, J.A., Ortega-Lara, A., Usma, J., Galvis, G., Villa-Navarro, F., Vásquez, L., Prada-Predreros, S., Ardila, C. (2005). Peces de los Andes de Colombia. Instituto de Investigación de Recursos Biológicos Alexander von Humboldt. Bogotá, DC, Colombia. p. 346.

Martínez, J., Cadena, D., Torres, M. (2016). Critical thermal limits of Poecilia caucana (Steindachner, 1880) (Cyprinodontiformes: Poeciliidae). Neotropical Ichthyology. 14 (1): 150-171.

Mercer, E., Mercer, T., Sayok, A. (2014). Effects of forest conversions to oil palm plantations on freshwater macroinvertebrates: A case study from Sarawak, Malaysia. Journal of Land Use Science. 9 (3): 260-277.

Montoya, D. (2014). Diversidad, estructura y relaciones ecomorfológicas de la ictiofauna en ecositemas lóticos del Bosque Seco Tropical, Tolima, Colombia. (Trabajo de Pregrado). Universidad del Tolima, Facultad de Ciencias, Departamento de Biología, Ibagué.

Pareja-Cardona, M., Jiménez-Segura, L., Villa- Navarro, F., Reinoso-Flórez, G., Gualtero-Lea, D., Ángel-Rojas, V. (2014). Áreas de reproducción de peces migratorios en la cuenca alta del río Magdalena, Colombia. Biota Colombiana. 15 (2): 40-53.

Pérez, M., Prada-Pedreros, S. (2015). Relationship between fish community and riparian vegetation cover in hydrological periods (Coffee-growing region, Colombia). Universitas Scientiarum. 16 (2): 119-139.

Posada, J., Roldán, G., Ramírez, J. (2000). Caracterización fisicoquímica y biológica de la calidad de aguas de la cuenca de la quebrada Piedras Blancas, Antioquia, Colombia. Revista de Biologia Tropical. 48 (1), 59-70.

Pusey, B., Arthington, A. (2003). Importance of the riparian zone to the conservation and management of freshwater fish: A review. Marine and Freshwater Research. 54: 1-16.

Provenzano, F.R., Villla- Navarro, F.A. (2017). A new species of the catfish genus Cordylancistrus (Siluriformes, Loricariidae) from the Magdalena River, Tolima, Colombia. Zootaxa. 4294 (5): 522-530.

Shimano, Y., Juen, L. (2016). How oil palm cultivation is affecting mayfly assemblages in Amazon streams? Annales de Limnologie - International Journal of Limnology. 52: 35-45.

Sweeney, B., Bott, T., Jackson, J., Kaplan, L., Newbold, J., Standley, L., Hession, W., Horwitz, R. (2004). Riparian deforestation, stream narrowing, and loss of stream ecosystem services. Proceedings of the National Academy of Sciences of the United States of America. 101 (39): 14132-14137.

Taphorn, D., Armbruster, J., Villa-Navarro, F., Keith, C. 2013. Trans-Andean Ancistrus (Siluriformes: Loricariidae). Zootaxa. 3641 (4): 343-370

Thioulouse J, Chessel, D. (1992). A Method for Reciprocal Scaling of Species Tolerance and Sample Diversity. Ecology. 73 (2): $670-80$.

Velázquez E, Gómez-Sal, A. (2009). Changes in the Herbaceous Communities on the Landslide of the Casita Volcano, Nicaragua, during Early Succession. Folia Geobotanica. 44: $1-18$

Villa-Navarro, F., García, L., Brínez, N., Zúñiga, P. (2004). Biodiversidad de la cuenca del río Coello. Biodiversidad Regional Fase I. Fecha de consulta: 10 de noviembre de 2017. Disponible en: http://www.cortolima.gov.co 
Villa-Navarro, F.A., Zúñiga-Upegui, P., Castro-Roa, D., García, J., García, L., Herrada-Yara, M. (2006). Peces del alto Magdalena, cuenca del río Magdalena, Colombia. Biota Colombiana. 7 (1): 3-22.

Villa-Navarro, F.A., García, L., Herrada, M., Lozano, Y. (2007). Biodiversidad de la cuenca del río Totare. Biodiversidad Regional Fase III. Fecha de consulta: 5 de octubre de 2017. Disponible en: http://www.cortolima.gov

Vieira, D., Shibatta, O. (2007). Peixes como indicadores da qualidade ambiental do ribeirão Esperança, município de Londrina, Paraná, Brasil. Biota Neotropica. 7 (1): 57-65.

Winemiller, K., Agostinho, A., Caramaschi, E. (2007). Fish ecology in tropical stream. En: Dudgeon, D., Tropical Stream Ecology (270). Elsevier Inc. p.107-146.

Zúñiga-Upegui, P., Villa-Navarro, F.A., Reinoso-Flórez, G., Ortega-Lara, A. (2005). Relación longitud-peso y frecuencia de tallas para los peces del género Chaetostoma (Siluriformes, Loricariidae), de la cuenca del río Coello, Colombia. Dahlia. 8: $47-52$.
Zúñiga-Upegui, P., Villa-Navarro, F. A., García-Melo, L., García-Melo, J., Reinoso-Flórez, G., Gualtero-Leal, D. y Ángel-Rojas, V. (2014). Aspectos ecológicos de Chaetostoma sp. (Siluriformes: Loricariidae) en el alto río Magdalena, Colombia. Biota Colombiana. 15 (2): 81-94.

Zúñiga-Upegui, P. (2005). Diversidad, distribución y algunos aspectos ecológicos de la familia Loricariidae (Pisces, Siluroidei) en la Cuenca del río Coello-Tolima (Colombia). (Trabajo de Pregrado). Universidad del Tolima, Facultad de Ciencias, Departamento de Biología, Ibagué.

Zuluaga, M., Fitzgerald, D., Giarrizzo, T., Winemiller, K. (2016). Morphologic and trophic diversity of fish assemblages in rapids of the Xingú River, a major Amazon tributary and region of endemism. Environmental Biology of Fishes. 99 (8-9): 647-658. 